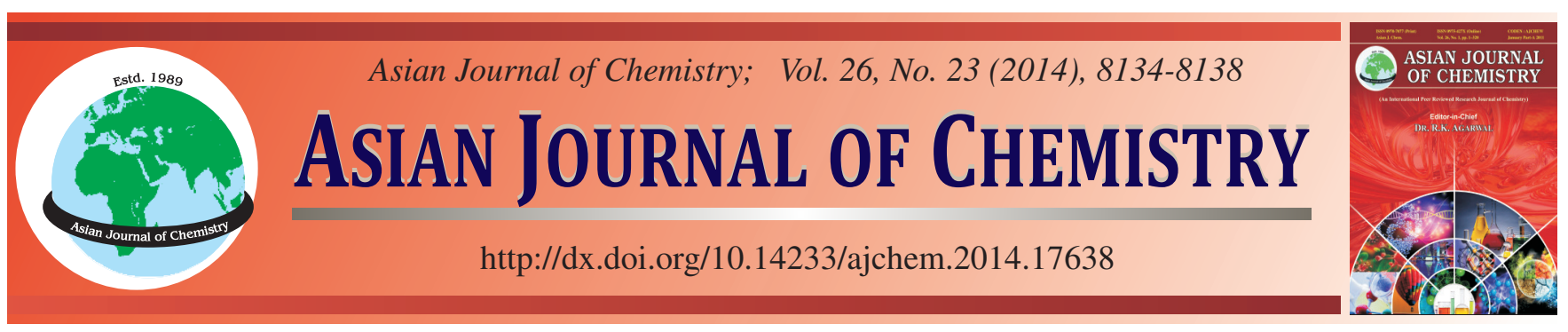

\title{
Synthesis, Characterization and Antimicrobial Activity of Long Chain Fatty Alkenoates of Metronidazole and their Novel Tetrazole Derivatives
}

\author{
Humaira Parveen $^{1, *}$, Sayeed Mukhtar ${ }^{1}$, Nadia Hussein El Sayed ${ }^{1}$ and Faisal Hayat ${ }^{2}$
}

${ }^{1}$ Department of Chemistry, Faculty of Science, University of Tabuk, Tabuk, Kingdom of Saudi Arabia

${ }^{2}$ College of Pharmacy and Division of Life and Pharmaceutical Sciences, Ewha Womans University, Seoul 120-750, Republic of Korea

*Corresponding author: Tel: +966 1442324 112/1071; E-mail: humaira_chem@yahoo.co.in

Keywords: Fatty alkenoates, Metronidazole, Tetrazole derivatives, Antimicrobial activity.

ᄂ - - - - - - - - - - - - - - - - - - - - - - - - - - - - -

\section{INTRODUCTION}

Infectious diseases caused by microbes such as bacteria and fungi are one of the leading causes of morbidity and mortality and the major reason for the increase in microbial infections is the resistance developed by these microbial organisms, particularly Gram-positive bacteria Staphylococcus aureus towards antimicrobial agents ${ }^{1}$. Therefore it is very necessary to seek for new drugs attacking crucial targets in the microbial pathogen in order to combat and relieve this tremendous prevalence. The modification of drug or prodrug is one approach that can lead both to prolong pharmacological activity and reduce adverse effects. Many seed oils, fatty acids and their derivatives are known for their antimicrobial ${ }^{2,3}$, antifungal $^{4}$ and pesticidal ${ }^{5}$ activities. A number of investigations have demonstrated that various fatty acid derivatives are promising molecules in cancer prevention and have potential in the treatment of cancers ${ }^{6-8}$. Recently fatty acid ester analogs have been found to be associated with diverse biological activities such as antioxidant ${ }^{9}$, antifeedant ${ }^{10}$, antiinflammatory $^{11}$, antiparasitic ${ }^{12}$, neuroprotective ${ }^{13}$ and antimicrobial ${ }^{14}$. Some fatty acid esters have been also found very effective for the treatment of dermatitis ${ }^{15}$, cardiovascular, hepatic and renal disorder ${ }^{16}$. Thus fatty acid derivatives may lead to a new route to potential pharmaceutical molecules. Moreover, tetrazole derivatives have also been reported to possess a broad spectrum of biological activities ${ }^{17-23}$. The purpose of this study was to find the novel bioactivity of various fatty acid derivatives of metronidazole. In view of these observations and as a part of our ongoing program devoted to the synthesis of diverse heterocycles, we had previously reported bisdioxazole derivatives ${ }^{24}, 2,4,6$-trisubstituted bis-pyrimidine derivatives $^{25}$ and 6-ferrocenyl-4-aryl-2-substituted pyrimidine derivatives ${ }^{26}$. In this study we report herein synthesis, characterization and in vitro antimicrobial activity of long chain fatty alkenoates of metronidazole and their $\alpha$-bromo-5'-methyl tetrazole derivatives.

\section{EXPERIMENTAL}

All chemicals, Undec-10-enoic acid (1) and (9Z)-octadec9-enoic acid (2) were purchased from Sigma-Aldrich Chemical Company (USA). Precoated aluminum sheets (silica gel 60 $\mathrm{F}_{254}$, Merck Germany) were used for thin-layer chromatography (TLC) and spots were visualized under UV light. Elemental analyses were performed on Heraeus Vario EL III analyzer. The results were within $\pm 0.3 \%$ of the theoretical values. IR spectra were recorded on Perkin-Elmer model 1600 FT-IR RX1 spectrophotometer as KBr discs. ${ }^{1} \mathrm{H}$ NMR and ${ }^{13} \mathrm{C}$ NMR spectra were recorded on Bruker AVANCE 400 spectrometer using $\mathrm{CDCl}_{3}$ as solvent with TMS as internal standard. ESI-MS was recorded on a MICROMASS QUATTRO II triple quadrupole mass spectrometer.

Long chain fatty alkenoates (5-8) of metronidazole were synthesized via N,N'-dicyclohexylcarbodiimide (DCC)mediated esterification ${ }^{27}$. A solution of long chain olefinic fatty 
acid, DCC and metronidazole in chloroform with catalytic amount of 4-(N,N-dimethylamino)pyridine (DMAP) was stirred mechanically at room temperature until esterification was complete (Scheme-I). (9Z, 12R)-12-hydroxyoctadec-9enoic (ricinoleic,) acid (3) and (9R, 12Z)- 9-hydroxyoctadec12-enoic (isoricinoleic,) acid (4) were isolated from Ricinus communis and Wrightia tinctoria seed oils, respectively following Gunstone's partition method ${ }^{28}$. The $\alpha$-bromo-5'methyl tetrazole derivatives of long chain fatty alkenoates of metronidazole were synthesized by the reaction of 2-(2methyl-5-nitro- $1 H$-imidazol-1-yl)-ethyl-undec-10-enoate (5) with bromine, acetonitrile and sodium azide in the presence of anhydrous aluminum chloride to afford the major product, 2-(2-methyl-5-nitro-1H-imidazol-1-yl)-ethyl 11-bromo-10(5'-methyl-1H-tetrazol-1-yl) undecanoate (5a) along with 2(2-methyl-5-nitro-1H-imidazol-1-yl)-ethyl 10,11-dibromo undecanoate $(\mathbf{5 b})$. Under similar reaction conditions 2-(2methyl-5-nitro- $1 H$-imidazol-1-yl)-ethyl-octadec-9-enoate (6) and 2-(2-methyl-5-nitro-1H-imidazol-1-yl)-ethyl-12hydroxyoctadec- 9-enoate (7) yielded 2-(2-methyl-5-nitro- $1 \mathrm{H}$ imidazol-1-yl)-ethyl-threo-9/10-bromo-10/9-(5'-methyl-1Htetrazol-1-yl) octadecanoate (6a) and 2-(2-methyl-5-nitro- $1 H$ imidazol-1-yl)-ethyl-threo-12-hydroxy-9/10-bromo-10/9-(5'methyl-1H-tetrazol-1-yl) octadecanoate (7a), respectively in addition to their dibromides (Z)-2-(2-methyl-5-nitro- $1 H$ imidazol-1-yl)-ethyl 9,10-dibromooctadec-9-enoate (6b) and 2-(2-methyl-5-nitro-1H-imidazol-1-yl)-ethyl-12-hydroxy9,10-dibromo-(5'-methyl-1H-tetrazol-1-yl) octadecanoate (7b), respectively (Scheme-II, III). The confirmation of $\mathbf{6 a}$ and $7 \mathbf{a}$ was designated on the basis of literature report and mechanistic approach ${ }^{29}$. All the synthesized compounds (5-8), (5a-7a) and (5b-7b) were well characterized by spectroscopic methods such as IR, ${ }^{1} \mathrm{H}$ NMR, ${ }^{13} \mathrm{C}$ NMR and ESI-MS and their purity was confirmed by elemental analysis.

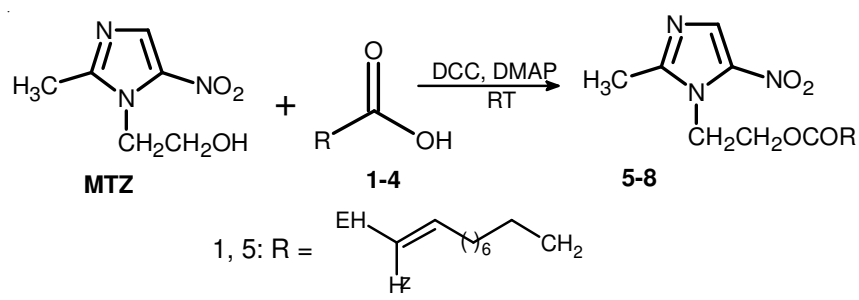

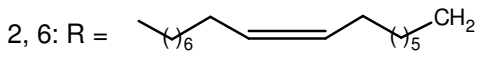<smiles>CCCC=CCC(O)CC=C=[Pt]</smiles>

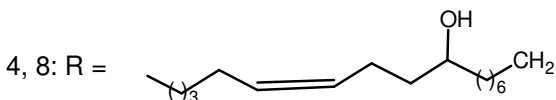

Scheme-I: General synthesis of long chain fatty alkenoates of metronidazole (5-8)

General procedure for the preparation of long chain fatty alkenoates (5-8): A solution of fatty acid $(0.01 \mathrm{~mol})$, DCC (0.011 mol), metronidazole $(0.01 \mathrm{~mol})$ and DMAP $(0.01$ $\mathrm{mol})$ in chloroform $(80 \mathrm{~mL})$ was stirred mechanically at room temperature until esterification was complete. The N,N'-

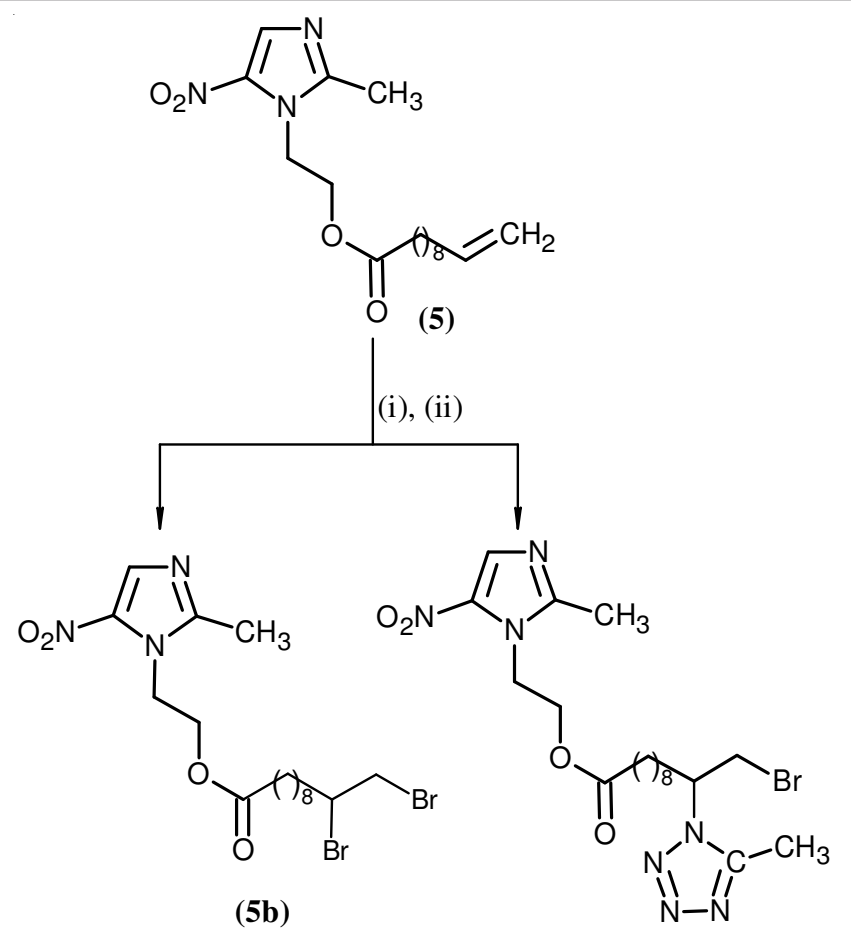

(5a)

Scheme-II: Synthesis of $\alpha$-bromo-5'-methyl tetrazole derivative of MTZ (5a) major product and $(\mathbf{5 b})$ minor product<smiles>[R]/C=C\CC(=O)OCCn1c([N+](=O)[O-])cnc1C</smiles>

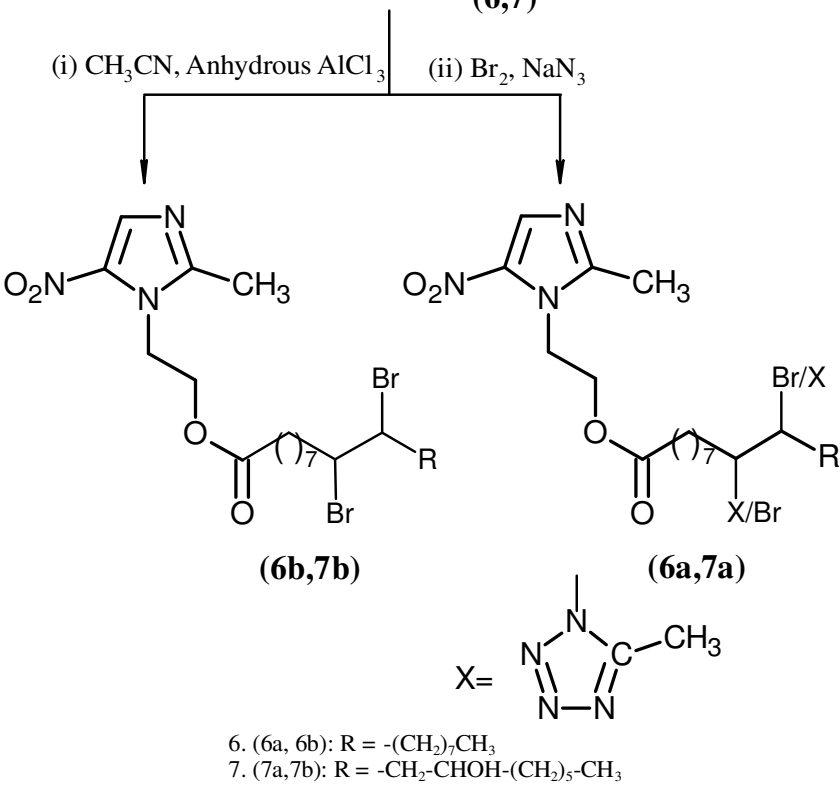

Scheme-III: Synthesis of $\alpha$-bromo-5'-methyl tetrazole derivatives of MTZ $(6 \mathbf{a}$ and $7 \mathbf{a})$ major products and $(\mathbf{6 b}$ and $7 \mathbf{b})$ minor products

dicyclohexylurea was filtered off and the filtrate was washed with water $(3 \times 100 \mathrm{~mL}), 5 \%$ acetic acid solution $(3 \times 100 \mathrm{~mL})$ again with water $(3 \times 100 \mathrm{~mL})$ and then dried over anhydrous 
sodium sulphate. The solvent was removed under reduced pressure to give a liquid residue. The latter was passed through a column of silica gel (230-400 mesh) and eluted with chloroform-ethyl acetate (95:5) to yield pure compound.

2-(2-Methyl-5-nitro-1H-imidazol-1-yl)-ethyl-undec10-enoate (5): Yield $95 \%$; colourless liquid; Anal. calc. for $\mathrm{C}_{17} \mathrm{H}_{27} \mathrm{~N}_{3} \mathrm{O}_{4}$ : C 60.51, H 8.07, N $12.45 \%$. Found: $\mathrm{C} 60.53 ; \mathrm{H}$ 8.05, N $12.41 \%$. IR $\left(\mathrm{KBr}, \mathrm{v}_{\max }, \mathrm{cm}^{-1}\right): 1738(\mathrm{C}=\mathrm{O}), 2926$, $2854(\mathrm{C}-\mathrm{H}), 1530,1465(\mathrm{C}=\mathrm{C}), 1533\left(\mathrm{NO}_{2}\right) ;{ }^{1} \mathrm{H} \mathrm{NMR}\left(\mathrm{CDCl}_{3}\right)$ $\delta(\mathrm{ppm}): 7.88(\mathrm{~s}, 1 \mathrm{H}, \mathrm{N}-\mathrm{CH}=\mathrm{C}), 2.11\left(\mathrm{t}, 2 \mathrm{H}, \mathrm{N}-\mathrm{CH}_{2}\right), 1.5(\mathrm{t}$, $\left.2 \mathrm{H}, \mathrm{N}-\mathrm{CH}_{2}-\mathrm{CH}_{2}\right), 2.45\left(\mathrm{~s}, 3 \mathrm{H}, \mathrm{C}-\mathrm{CH}_{3}\right), 5.75\left(\mathrm{~m}, 1 \mathrm{H}, \mathrm{CH}_{2}=\right.$ $\mathrm{CH}), 4.86\left(\mathrm{dd}, 1 \mathrm{H}, \mathrm{H}_{\mathrm{Z}} \mathrm{C}=\mathrm{CH}, J_{\mathrm{HZ}-\mathrm{H}}=10, J_{\mathrm{HZ}-\mathrm{HE}}=1.6 \mathrm{~Hz}\right)$, $4.92\left(\mathrm{dd}, 1 \mathrm{H}, \mathrm{H}_{\mathrm{E}} \mathrm{C}=\mathrm{CH}, J_{\mathrm{HE}-\mathrm{H}}=17.2 \mathrm{~Hz}, J_{\mathrm{HE}-\mathrm{Hz}}=1.6 \mathrm{~Hz}\right)$, 1.99 (t, 2H, CO-CH $\mathrm{CH}_{2}, 1.30$ (m, 2H, CO- $\mathrm{CH}_{2}-\mathrm{CH}_{2}$ ), 1.2 (br s, chain $\left.\mathrm{CH}_{2}\right),{ }^{13} \mathrm{C} \mathrm{NMR}\left(\mathrm{CDCl}_{3}\right) \delta(\mathrm{ppm}): 173.8(\mathrm{C}=\mathrm{O}), 150.8$ $(\mathrm{N}=C-\mathrm{N}), 133.0(\mathrm{~N}-C=\mathrm{C}), 139.1,(\mathrm{O} 2 \mathrm{~N}-C), 14.1\left(\mathrm{C}-\mathrm{CH}_{3}\right), 35.8$ $\left(\mathrm{N}-\mathrm{CH}_{2}\right), 62.3\left(\mathrm{~N}-\mathrm{CH}_{2}-\mathrm{CH}_{2}\right), 33.9\left(\mathrm{CO}-\mathrm{CH}_{2}\right), 24.6\left(\mathrm{CO}-\mathrm{CH}_{2}-\right.$ $\left.\mathrm{CH}_{2}\right), 29.0,30.6,29.3,29.6,29.7,33.8,138.5\left(\mathrm{CH}_{2}=\mathrm{CH}\right)$, $114.1\left(\mathrm{CH}_{2}=\mathrm{CH}\right)$, ESI-MS $m / z:\left[\mathrm{M}^{+}+1\right] 338.41$.

2-(2-Methyl-5-nitro-1H-imidazol-1-yl)-ethyl-octadec9-enoate (6): Yield $95 \%$; pale yellow liquid; Anal. calc. for $\mathrm{C}_{24} \mathrm{H}_{41} \mathrm{~N}_{3} \mathrm{O}_{4}$ : C 66.17, H 9.49, N 09.65 \%. Found: C 66.20; $\mathrm{H}$ 9.51, N $09.61 \%$. IR $\left(\mathrm{KBr}, \mathrm{v}_{\max }, \mathrm{cm}^{-1}\right): 1739(\mathrm{C}=\mathrm{O}), 2924$, $2853(\mathrm{C}-\mathrm{H}), 1531,1465(\mathrm{C}=\mathrm{C}), 1533\left(\mathrm{NO}_{2}\right) ;{ }^{1} \mathrm{H} \mathrm{NMR}\left(\mathrm{CDCl}_{3}\right)$ $\delta$ (ppm): $7.93(\mathrm{~s}, 1 \mathrm{H}, \mathrm{N}-\mathrm{CH}=\mathrm{C}), 4.56\left(\mathrm{t}, 2 \mathrm{H}, \mathrm{N}-\mathrm{CH}_{2}\right), 4.37(\mathrm{t}$, $\left.2 \mathrm{H}, \mathrm{N}-\mathrm{CH}_{2}-\mathrm{CH}_{2}\right), 2.49$ (s, 3H, C- $\left.\mathrm{CH}_{3}\right), 5.31(\mathrm{~m}, 2 \mathrm{H}, \mathrm{CH}=\mathrm{CH}$ ), $2.24\left(\mathrm{~m}, 2 \mathrm{H}, \mathrm{CO}-\mathrm{CH}_{2}\right), 1.68\left(\mathrm{~m}, 2 \mathrm{H}, \mathrm{CO}-\mathrm{CH}_{2}-\mathrm{CH}_{2}\right), 1.23$ (br $\mathrm{s}, 2 \times 9 \mathrm{H}), 1.55\left(\mathrm{~m}, 4 \mathrm{H}, \mathrm{CH}_{2}-\mathrm{CH}_{2}-\mathrm{CH}=\mathrm{CH}-\mathrm{CH}_{2}-\mathrm{CH}_{2}\right), 1.99$ (m, 4H, $\left.\mathrm{CH}_{2}-\mathrm{CH}=\mathrm{CH}-\mathrm{CH}_{2}\right), 1.30\left(\mathrm{~m}, 2 \mathrm{H}, \mathrm{CH}_{2} \mathrm{CH}_{3}\right), 0.89$ (t, $3 \mathrm{H}$, terminal $\left.\mathrm{CH}_{3}\right),{ }^{13} \mathrm{C} \mathrm{NMR}\left(\mathrm{CDCl}_{3}\right) \delta(\mathrm{ppm}): 173.1(\mathrm{C}=\mathrm{O})$, $150.7(\mathrm{~N}=C-\mathrm{N}), 132.7(\mathrm{~N}-C=\mathrm{C}), 138.5\left(\mathrm{O}_{2} \mathrm{~N}-C\right), 14.1\left(\mathrm{C}-\mathrm{CH}_{3}\right)$, $45.1\left(\mathrm{~N}-\mathrm{CH}_{2}\right), 62.3\left(\mathrm{~N}-\mathrm{CH}_{2}-\mathrm{CH}_{2}\right), 33.9\left(\mathrm{CO}-\mathrm{CH}_{2}\right), 25.5(\mathrm{CO}-$ $\left.\mathrm{CH}_{2}-\mathrm{CH}_{2}\right)$, 29.0, 29.1, 29.3, 30.8, 27.2, 130.0, 129.6, 27.1, $30.9,29.5,29.6,29.7,33.6,31.8,22.8$. ESI-MS m/z: $\left[\mathrm{M}^{+}+1\right]$ 436.6 .

2-(2-Methyl-5-nitro-1H-imidazol-1-yl)-ethyl-12hydroxyoctadec-9-enoate (7): Yield $90 \%$; yellow liquid; Anal. calc. for $\mathrm{C}_{24} \mathrm{H}_{41} \mathrm{~N}_{3} \mathrm{O}_{5}$ : C 63.83, H 9.15, N 09.30\%. Found: C 63.80; H 9.12, N 09.34\%. IR (KBr, $\left.v_{\max }, \mathrm{cm}^{-1}\right): 1739(\mathrm{C}=\mathrm{O})$, $3352(\mathrm{OH}), 2960,2853(\mathrm{C}-\mathrm{H}), 1534,1465(\mathrm{C}=\mathrm{C}), 1534\left(\mathrm{NO}_{2}\right)$; ${ }^{1} \mathrm{H} \mathrm{NMR}\left(\mathrm{CDCl}_{3}\right) \delta(\mathrm{ppm}): 7.85(\mathrm{~s}, 1 \mathrm{H}, \mathrm{N}-\mathrm{CH}=\mathrm{C}), 4.54(\mathrm{t}$, $2 \mathrm{H}, \mathrm{N}-\mathrm{CH}_{2}$ ), 4.33 (t, 2H, N-CH $-\mathrm{CH}_{2}$ ), 2.45 (s, 3H, C-CH $\mathrm{CH}_{3}$, 5.56-5.39 (m, 2H, $\mathrm{CH}=\mathrm{CH}), 3.54(\mathrm{~m}, 1 \mathrm{H}, \mathrm{CH}-\mathrm{OH}), 3.35(\mathrm{br}$ $\mathrm{s}, 1 \mathrm{H}, \mathrm{CH}-\mathrm{OH}) 1.99$ (m, 2H, C-11), 2.01 (m, 2H, C-8), 2.28 (m, 2H, CO- $\mathrm{CH}_{2}$ ), 1.62 (m, 2H, CO- $\left.\mathrm{CH}_{2}-\mathrm{CH}_{2}\right), 1.28$ (br s, $2 \times$ $9 \mathrm{H}), 1.99\left(\mathrm{~m}, 4 \mathrm{H}, \mathrm{CH}_{2}-\mathrm{CH}=\mathrm{CH}-\mathrm{CH}_{2}\right), 1.30\left(\mathrm{~m}, 2 \mathrm{H}, \mathrm{CH}_{2} \mathrm{CH}_{3}\right)$, 0.90 (distorted t, $3 \mathrm{H}$, terminal $\left.\mathrm{CH}_{3}\right),{ }^{13} \mathrm{C} \mathrm{NMR}\left(\mathrm{CDCl}_{3}\right) \delta(\mathrm{ppm})$ : $173.2(C=\mathrm{O}), 152.5(\mathrm{~N}=C-\mathrm{N}), 133.0(\mathrm{~N}-C=\mathrm{C}), 140.5\left(\mathrm{O}_{2} \mathrm{~N}-\right.$ $C), 34.8\left(\mathrm{~N}-\mathrm{CH}_{2}\right), 62.5\left(\mathrm{~N}-\mathrm{CH}_{2}-\mathrm{CH}_{2}\right), 34.9\left(\mathrm{CO}-\mathrm{CH}_{2}\right), 24.9$ (CO-CH $2-C H 2)$, 29.1, 29.5, 131.8 (C-9), 131.5 (C-10), 27.3 (C-8), 27.4 C-11), 72.8 (C-12), 34.6 (C-13), 29.7, 31.7, 31.8, 22.8, 32.2, 36.4, 14.1, 12.0. ESI-MS $m / z:\left[\mathrm{M}^{+}+1\right] 452.6$.

2-(2-Methyl-5-nitro-1H-imidazol-1-yl)-ethyl-9hydroxyoctadec-12-enoate (8): Yield $92 \%$; yellow liquid; Anal. calc. for $\mathrm{C}_{24} \mathrm{H}_{41} \mathrm{~N}_{3} \mathrm{O}_{5}$ : C 63.83, H 9.15, N 09.30\%. Found: C 63.80; H 9.12, N 09.34\%. IR ( KBr, $\left.v_{\max }, \mathrm{cm}^{-1}\right): 1737(\mathrm{C}=\mathrm{O})$, $3352(\mathrm{OH}), 2960,2853(\mathrm{C}-\mathrm{H}), 1534,1465(\mathrm{C}=\mathrm{C}), 1534\left(\mathrm{NO}_{2}\right)$; ${ }^{1} \mathrm{H} \mathrm{NMR}\left(\mathrm{CDCl}_{3}\right) \delta(\mathrm{ppm}): 7.83(\mathrm{~s}, 1 \mathrm{H}, \mathrm{N}-\mathrm{CH}=\mathrm{C}), 4.63(\mathrm{t}$, $\left.2 \mathrm{H}, \mathrm{N}-\mathrm{CH}_{2}\right), 4.37$ (t, $\left.2 \mathrm{H}, \mathrm{N}-\mathrm{CH}_{2}-\mathrm{CH}_{2}\right), 2.44$ (s, 3H, C-CH $\mathrm{CH}_{3}$, $5.37(\mathrm{~m}, 2 \mathrm{H}, \mathrm{CH}=\mathrm{CH}), 3.35(\mathrm{~m}, 1 \mathrm{H}, \mathrm{CH}-\mathrm{OH}), 3.30$ (br s, $1 \mathrm{H}$, CH-OH) 2.10 (m, 2H, C-11), 2.02 (m, 2H, C-14), 2.28 (m, $2 \mathrm{H}, \mathrm{CO}-\mathrm{CH}_{2}$ ), 1.65 (m, 2H, CO-CH $-\mathrm{CH}_{2}$ ), 1.29 (br s, $2 \times$ 9H), $1.30\left(\mathrm{~m}, 2 \mathrm{H}, \mathrm{CH}_{2} \mathrm{CH}_{3}\right), 0.89$ (distorted t, $3 \mathrm{H}$, terminal $\left.\mathrm{CH}_{3}\right),{ }^{13} \mathrm{C} \mathrm{NMR}\left(\mathrm{CDCl}_{3}\right) \delta(\mathrm{ppm}): 173.0(\mathrm{C}=\mathrm{O}), 154.2(\mathrm{~N}=\mathrm{C}-$ $\mathrm{N}), 138.2(\mathrm{~N}-C=\mathrm{C}), 140.0\left(\mathrm{O}_{2} \mathrm{~N}-C\right), 35.1\left(\mathrm{~N}-\mathrm{CH}_{2}\right), 62.5(\mathrm{~N}-$ $\left.\mathrm{CH}_{2}-\mathrm{CH}_{2}\right), 33.6\left(\mathrm{CO}-\mathrm{CH}_{2}\right), 25.1\left(\mathrm{CO}-\mathrm{CH}_{2}-\mathrm{CH}_{2}\right), 29.0,29.5$, 29.9, 24.1, 36.8 (C-8), 72.3 (C-9), 38.4 (C-10), 26.5, 131.8 (C-12), 131.5 (C-13), 30.6, 29.5, 31.7, 22.6, 14.0, 12.4. ESI$\mathrm{MS} m / z:\left[\mathrm{M}^{+}+1\right] 452.6$.

General procedure for the synthesis of $\alpha$-bromo-5methyl tetrazoles (5a-7a)): To the ice-cooled solution of fatty alkenoates $(\mathbf{5}-7)(10 \mathrm{mmol})$ in acetonitrile $(50 \mathrm{~mL})$ anhydrous aluminum chloride $(10 \mathrm{mmol})$ was added. Then the bromine $(10 \mathrm{mmol})$ was added to the above cooled $\left(0^{\circ} \mathrm{C}\right)$ and well stirred mixture, followed by addition of sodium azide $(10 \mathrm{mmol})$ in portions. After that the reaction mixture was allowed to attain room temperature and stirred for 6-8 h then filtered and the filtrate diluted with water and extracted with chloroform (100 $\mathrm{mL} \times 4)$. The extract was washed with water and dried over anhydrous sodium sulphate. The solvent was removed under reduced pressure to give the crude product which was chromatographed over a column of silica gel using hexane with increasing amount of chloroform as eluent to isolate $\mathbf{5 a - 7 a}$ as major products and $\mathbf{5 b} \mathbf{b} \mathbf{- 7} \mathbf{b}$ as minor products.

2-(2-Methyl-5-nitro-1H-imidazol-1-yl)-ethyl-11bromo-10-(5'-methyl-1H-tetrazol-1-yl)undecanoate (5a): Yield $65 \%$; dark brown liquid; Anal. calc. for $\mathrm{C}_{19} \mathrm{H}_{30} \mathrm{~N}_{6} \mathrm{O}_{4} \mathrm{Br}$ : C 46.92, H 6.22, Br 16.43, N 17.28 \%. Found: C 46.94; H 6.25, $\mathrm{N} 16.41 \%$. IR $\left(\mathrm{KBr}, \mathrm{v}_{\max }, \mathrm{cm}^{-1}\right): 1738(\mathrm{C}=\mathrm{O}), 2928,2854(\mathrm{C}-$ $\mathrm{H}), 1534\left(\mathrm{NO}_{2}\right), 1520,1365,1245,984,667 ;{ }^{1} \mathrm{H} \mathrm{NMR}\left(\mathrm{CDCl}_{3}\right)$ $\delta$ (ppm): 7.87 ( s, $1 \mathrm{H}, \mathrm{N}-\mathrm{CH}=\mathrm{C}), 4.52\left(\mathrm{t}, 2 \mathrm{H}, \mathrm{N}-\mathrm{CH}_{2}\right), 4.30(\mathrm{t}$, $\left.2 \mathrm{H}, \mathrm{N}-\mathrm{CH}_{2}-\mathrm{CH}_{2}\right), 4.65$ (m, 1H, N-CH), 3.90 (s, 1H, $\mathrm{H}_{\mathrm{Z}} \mathrm{C}-\mathrm{Br}$ ), 3.8 (distorted d, $1 \mathrm{H}, H_{\mathrm{E}} \mathrm{C}-\mathrm{Br}, J=3.0 \mathrm{~Hz}$ ), 2.51 (s, $3 \mathrm{H}, \mathrm{C}-\mathrm{CH}_{3}$, tetrazole ring), $2.412\left(\mathrm{~s}, 3 \mathrm{H}, \mathrm{C}-\mathrm{CH}_{3}\right.$, imidazole ring), 2.18 (m, $2 \mathrm{H}, \mathrm{BrCH}_{2}-\mathrm{CH}-\mathrm{CH}_{2}$ ), 2.32 (distorted t, $2 \mathrm{H}, \mathrm{CO}-\mathrm{CH}_{2}$ ), 1.62 (m, 2H, CO- $\left.\mathrm{CH}_{2}-\mathrm{CH}_{2}\right), 1.29$ (br s, chain $\left.\mathrm{CH}_{2}\right),{ }^{13} \mathrm{CNMR}\left(\mathrm{CDCl}_{3}\right)$ $\delta(\mathrm{ppm}): 173.2(C=\mathrm{O}), 151.0(\mathrm{~N}=C-\mathrm{N}$, imidazole $), 132.3$ $(\mathrm{N}-C=\mathrm{C}), 150.1(\mathrm{~N}-C=\mathrm{N}$, tetrazole $), 140.0,\left(\mathrm{O}_{2} \mathrm{~N}-C\right), 14.5$ $\left(\mathrm{C}-\mathrm{CH}_{3}\right), 36.2\left(\mathrm{~N}-\mathrm{CH}_{2}\right), 62.4\left(\mathrm{~N}-\mathrm{CH}_{2}-\mathrm{CH}_{2}\right), 33.0\left(\mathrm{CO}-\mathrm{CH}_{2}\right)$, $25.8\left(\mathrm{CO}-\mathrm{CH}_{2}-\mathrm{CH}_{2}\right), 28.9,28.6,28.7,25.7,24.6$, 30.0, 45.5, 34.8, 11.9 ESI-MS $m / z:\left[\mathrm{M}^{+}+1\right] 487.38$.

2-(2-Methyl-5-nitro-1H-imidazol-1-yl)-ethyl 10,11dibromoundecanoate (5b): Yield 15\%; dark brown liquid; Anal. calc. for $\mathrm{C}_{17} \mathrm{H}_{27} \mathrm{~N}_{3} \mathrm{O}_{4} \mathrm{Br}_{2}$ : C 41.06, $\mathrm{H}$ 5.47, $\mathrm{Br} 32.14, \mathrm{~N}$ $8.45 \%$. Found: C 41.09; H 5.45, N 8.42\%. IR ( KBr, $\left.v_{\max }, \mathrm{cm}^{-1}\right)$ : $1737.0(\mathrm{C}=\mathrm{O}), 2928,2852(\mathrm{C}-\mathrm{H}), 1533\left(\mathrm{NO}_{2}\right), 1529,1365$, 670; ${ }^{1} \mathrm{H}$ NMR $\left(\mathrm{CDCl}_{3}\right) \delta(\mathrm{ppm}): 7.82(\mathrm{~s}, 1 \mathrm{H}, \mathrm{N}-\mathrm{CH}=\mathrm{C}), 4.50$ (t, $\left.2 \mathrm{H}, \mathrm{N}-\mathrm{CH}_{2}\right), 4.31$ (t, $2 \mathrm{H}, \mathrm{N}-\mathrm{CH}_{2}-\mathrm{CH}_{2}$ ), 2.44 (s, 3H, C-CH imidazole ring), 3.58-4.40 (m, 3H, $\left.\mathrm{BrCH}_{2}-\mathrm{CH}-\mathrm{Br}\right), 2.01$ (m, $2 \mathrm{H}, \mathrm{Br}-\mathrm{CH}_{2}-\mathrm{CH}-\mathrm{Br}-\mathrm{CH}_{2}$ ), 2.32 (distorted t, $2 \mathrm{H}, \mathrm{CO}-\mathrm{CH}_{2}$ ), 1.62 (m, $\left.2 \mathrm{H}, \mathrm{CO}-\mathrm{CH}_{2}-\mathrm{CH}_{2}\right), 1.28$ (br s, chain $\left.\mathrm{CH}_{2}\right),{ }^{13} \mathrm{C} \mathrm{NMR}\left(\mathrm{CDCl}_{3}\right)$ $\delta(\mathrm{ppm}): 173.0(C=\mathrm{O}), 151.1(\mathrm{~N}=C-\mathrm{N}$, imidazole $), 133.0(\mathrm{~N}-$ $C=\mathrm{C}), 138.0,\left(\mathrm{O}_{2} \mathrm{~N}-\mathrm{C}\right), 14.2\left(\mathrm{C}-\mathrm{CH}_{3}\right), 36.9\left(\mathrm{~N}-\mathrm{CH}_{2}\right), 62.4(\mathrm{~N}-$ $\left.\mathrm{CH}_{2}-\mathrm{CH}_{2}\right), 34.5\left(\mathrm{CO}-\mathrm{CH}_{2}\right), 25.2\left(\mathrm{CO}-\mathrm{CH}_{2}-\mathrm{CH}_{2}\right), 28.1,28.4$, 28.7, 25.9, 36.6, 51.6, 37.1, 10.9 ESI-MS $m / z$ : [ $\left.\mathrm{M}^{+}+1\right] 498.22$.

2-(2-Methyl-5-nitro-1H-imidazol-1-yl)-ethyl-threo-9/ 10-bromo-10/9-(5'-methyl-1H-tetrazol-1-yl) octadecanoate (6a): Yield $72 \%$; brown liquid; Anal. calc. for $\mathrm{C}_{26} \mathrm{H}_{44} \mathrm{~N}_{7} \mathrm{O}_{4} \mathrm{Br}$ : 
C 52.17, H 7.41, Br 13.35, N $16.38 \%$. Found: C 52.20; H 7.45, Br 13.31, N 16.40\%. IR (KBr, $\left.v_{\max }, \mathrm{cm}^{-1}\right): 1737(\mathrm{C}=\mathrm{O})$, 2928, 2856 (C-H), 1533, 1523, 1366, 1245, 984, 667; ${ }^{1} \mathrm{H}$ NMR $\left(\mathrm{CDCl}_{3}\right) \delta(\mathrm{ppm}): 7.82(\mathrm{~s}, 1 \mathrm{H}, \mathrm{N}-\mathrm{CH}=\mathrm{C}), 4.53\left(\mathrm{t}, 2 \mathrm{H}, \mathrm{N}-\mathrm{CH}_{2}\right)$, 4.41 (t, $\left.2 \mathrm{H}, \mathrm{N}-\mathrm{CH}_{2}-\mathrm{CH}_{2}\right), 2.45$ (s, 3H, C-CH $\mathrm{CH}_{3}$, imidazole), 2.52 (s, 3H, C- $\mathrm{CH}_{3}$, tetrazole), $4.67(\mathrm{~m}, 2 \mathrm{H}, \mathrm{CH}=\mathrm{CH}), 2.31(\mathrm{~m}$, 2H, CO- $\mathrm{CH}_{2}$ ), 1.64 (m, 2H, CO- $\mathrm{CH}_{2}-\mathrm{CH}_{2}$ ), 1.29 (br s, chain $\left.\mathrm{CH}_{2}\right), 1.89\left(\mathrm{~m}, 4 \mathrm{H}, \mathrm{CH}_{2}-\mathrm{CH}=\mathrm{CH}-\mathrm{CH}_{2}\right), 1.32\left(\mathrm{~m}, 2 \mathrm{H}, \mathrm{CH}_{2} \mathrm{CH}_{3}\right)$, 0.88 (distorted t, $3 \mathrm{H}$, terminal $\left.\mathrm{CH}_{3}\right),{ }^{13} \mathrm{C} \mathrm{NMR}\left(\mathrm{CDCl}_{3}\right) \delta(\mathrm{ppm})$ : $173.1(C=\mathrm{O}), 151.2(\mathrm{~N}=C-\mathrm{N}), 152.4(\mathrm{~N}-C=\mathrm{N}$, tetrazole $), 129.9$ $(\mathrm{N}-C=\mathrm{C}), 141.0\left(\mathrm{O}_{2} \mathrm{~N}-\mathrm{C}\right), 14.0\left(\mathrm{C}-\mathrm{CH}_{3}\right), 35.6\left(\mathrm{~N}-\mathrm{CH}_{2}\right), 62.5$ $\left(\mathrm{N}-\mathrm{CH}_{2}-\mathrm{CH}_{2}\right), 34.9\left(\mathrm{CO}-\mathrm{CH}_{2}\right), 25.2\left(\mathrm{CO}-\mathrm{CH}_{2}-\mathrm{CH}_{2}\right), 29.1$, 29.5, 29.0, 24.9, 36.8, 36.7, 60.0, 60.2, 36.5, 24.8, 29.0, 29.4, 29.6, 31.7, 22.9, 14.5, ESI-MS $m / z:\left[\mathrm{M}^{+}+1\right] 599.58$.

(Z)-2-(2-Methyl-5-nitro-1H-imidazol-1-yl)-ethyl 9,10dibromooctadec-9-enoate (6b): Yield $16 \%$; brown liquid; Anal. calc. for $\mathrm{C}_{24} \mathrm{H}_{41} \mathrm{~N}_{3} \mathrm{O}_{4} \mathrm{Br}_{2}$ : C 48.41, $\mathrm{H}$ 6.94, $\mathrm{Br} 26.84, \mathrm{~N}$ $7.06 \%$. Found: C 48.40; H 6.98, Br 26.81, N 7.09\%. IR (KBr, $\left.\mathrm{V}_{\max }, \mathrm{cm}^{-1}\right): 1738(\mathrm{C}=\mathrm{O}), 2926,2850,1534,667 ;{ }^{1} \mathrm{H}$ NMR $\left(\mathrm{CDCl}_{3}\right) \delta$ (ppm): $7.88(\mathrm{~s}, 1 \mathrm{H}, \mathrm{N}-\mathrm{CH}=\mathrm{C}), 4.51\left(\mathrm{t}, 2 \mathrm{H}, \mathrm{N}-\mathrm{CH}_{2}\right)$, 4.32 (t, $\left.2 \mathrm{H}, \mathrm{N}-\mathrm{CH}_{2}-\mathrm{CH}_{2}\right), 2.44$ (s, 3H, C-CH $\mathrm{CH}_{3}$, imidazole), 2.52 (s, $3 \mathrm{H}, \mathrm{C}-\mathrm{CH}_{3}$, tetrazole), 4.31 (m, $\left.2 \mathrm{H}, \mathrm{Br}-\mathrm{CH}=\mathrm{CH}-\mathrm{Br}\right), 2.30$ (m, 2H, CO-CH $), 1.61$ (m, 2H, CO- $\mathrm{CH}_{2}-\mathrm{CH}_{2}$ ), 1.30 (br s, chain $\mathrm{CH}_{2}$ ), $1.98\left(\mathrm{~m}, 4 \mathrm{H}, \mathrm{CH}_{2}-\mathrm{CH}=\mathrm{CH}-\mathrm{CH}_{2}\right), 1.30(\mathrm{~m}, 2 \mathrm{H}$, $\mathrm{CH}_{2} \mathrm{CH}_{3}$ ), 0.89 (distorted t, $3 \mathrm{H}$, terminal $\mathrm{CH}_{3}$ ), ${ }^{13} \mathrm{C}$ NMR $\left(\mathrm{CDCl}_{3}\right) \delta(\mathrm{ppm}): 173.0(\mathrm{C}=\mathrm{O}), 151.2(\mathrm{~N}=C-\mathrm{N}), 130.9(\mathrm{~N}-$ $C=\mathrm{C}), 140.0\left(\mathrm{O}_{2} \mathrm{~N}-\mathrm{C}\right), 10.5 .0\left(\mathrm{C}-\mathrm{CH}_{3}\right), 35.5\left(\mathrm{~N}-\mathrm{CH}_{2}\right), 62.6(\mathrm{~N}-$ $\left.\mathrm{CH}_{2}-\mathrm{CH}_{2}\right), 34.9\left(\mathrm{CO}-\mathrm{CH}_{2}\right), 25.4\left(\mathrm{CO}-\mathrm{CH}_{2}-\mathrm{CH}_{2}\right), 29.3,29.5$, 29.4, 25.2, 32.8, 32.4, 57.9, 57.8, 25.5, 25.8, 29.2, 29.4, 30.9, 24.4, 14.5, ESI-MS m/z: $\left[\mathrm{M}^{+}+1\right]$ 596.41.

2-(2-Methyl-5-nitro-1H-imidazol-1-yl)-ethyl-threo-12hydroxy-9/10-bromo-10/9-(5'-methyl-1H-tetrazol-1-yl) octadecanoate (7a): Yield $68 \%$; brown liquid; Anal. calc. for $\mathrm{C}_{26} \mathrm{H}_{44} \mathrm{~N}_{7} \mathrm{O}_{5} \mathrm{Br}$ : C 50.81, H 7.22, Br 13.00, N $15.95 \%$. Found: C 50.80; H 7.25, Br 13.14, N 15.91\%. IR (KBr, $\left.v_{\max }, \mathrm{cm}^{-1}\right)$ : $1737(\mathrm{C}=\mathrm{O}), 3350(\mathrm{OH}), 2927,2853,1534,1461,1240,982$, 668; ${ }^{1} \mathrm{H}$ NMR $\left(\mathrm{CDCl}_{3}\right) \delta(\mathrm{ppm}): 7.87$ (s, 1H, N-CH=C), 4.52 (t, $\left.2 \mathrm{H}, \mathrm{N}-\mathrm{CH}_{2}\right), 4.30$ (t, 2H, N-CH $-\mathrm{CH}_{2}$ ), 2.45 (s, 3H, C-CH $\mathrm{CH}_{3}$, $5.37(\mathrm{~m}, 2 \mathrm{H}, \mathrm{CH}=\mathrm{CH}), 3.58(\mathrm{~m}, 1 \mathrm{H}, \mathrm{CH}-\mathrm{OH}), 2.54$ (s, 3H, C$\mathrm{CH}_{3}$, tetrazole), 2.30 (br s, $1 \mathrm{H}, \mathrm{CH}-\mathrm{OH}$ ), 2.32 (m, 2H, CO$\mathrm{CH}_{2}$ ), 1.63 (m, 2H, CO- $\mathrm{CH}_{2}-\mathrm{CH}_{2}$ ), 1.28 (br s, chain $\mathrm{CH}_{2}$ ), 2.19 and $2.03\left(\mathrm{~m}, 2 \mathrm{H}\right.$, each for $\left.\mathrm{CH}_{2}-\mathrm{CH}=\mathrm{CH}-\mathrm{CH}_{2}\right), 1.30(\mathrm{~m}, 2 \mathrm{H}$, $\mathrm{CH}_{2} \mathrm{CH}_{3}$ ), 0.89 (distorted t, $3 \mathrm{H}$, terminal $\mathrm{CH}_{3}$ ), ${ }^{13} \mathrm{C} \mathrm{NMR}$ $\left(\mathrm{CDCl}_{3}\right) \delta(\mathrm{ppm}): 173(C=\mathrm{O}), 152.0(\mathrm{~N}=C-\mathrm{N}), 154.5(\mathrm{~N}-C=\mathrm{N}$, tetrazole), $139.1(\mathrm{~N}-\mathrm{C}=\mathrm{C}), 140.2\left(\mathrm{O}_{2} \mathrm{~N}-\mathrm{C}\right), 34.5\left(\mathrm{~N}-\mathrm{CH}_{2}\right), 62.1$ $\left(\mathrm{N}-\mathrm{CH}_{2}-\mathrm{CH}_{2}\right), 33.4\left(\mathrm{CO}-\mathrm{CH}_{2}\right), 24.8\left(\mathrm{CO}-\mathrm{CH}_{2}-\mathrm{CH}_{2}\right), 29.1$, 29.6, 29.0, 25.4, 34.4 (C-8), 60.8 (C-9), 61.0 (C-10), 45.6 C11), 65.5 (C-12), 37.3 (C-13), 24.2, 28.6, 30.2, 24.0, 14.1, 12.0, ESI-MS $m / z:\left[\mathrm{M}^{+}+1\right] 615.58$.

2-(2-Methyl-5-nitro-1H-imidazol-1-yl)-ethyl-12hydroxy-9,10-dibromo-(5'-methyl-1H-tetrazol-1-yl) octadecanoate (7b): Yield $16 \%$; brown liquid; Anal. calc. for $\mathrm{C}_{24} \mathrm{H}_{41} \mathrm{~N}_{3} \mathrm{O}_{5} \mathrm{Br}_{2}$ : C 47.15, H 6.76, Br 26.14, N $6.87 \%$. Found: C 47.13; H 6.78, Br 26.13, N $6.83 \%$. IR (KBr, $v_{\max }$, $\left.\mathrm{cm}^{-1}\right): 1737(\mathrm{C}=\mathrm{O}), 3355(\mathrm{OH}), 2925,2853,1533,1461,1534$, 667; ${ }^{1} \mathrm{H}$ NMR $\left(\mathrm{CDCl}_{3}\right) \delta(\mathrm{ppm}): 7.87$ (s, 1H, N-CH=C), 4.51 (t, $\left.2 \mathrm{H}, \mathrm{N}-\mathrm{CH}_{2}\right), 4.32$ (t, 2H, N-CH $-\mathrm{CH}_{2}$ ), 2.44 (s, 3H, C-CH $\mathrm{CH}_{3}$, $5.29(\mathrm{~m}, 2 \mathrm{H}, \mathrm{CH}=\mathrm{CH}), 3.59$ (m, 1H, $\mathrm{CH}-\mathrm{OH}), 2.31$ (br s, $1 \mathrm{H}$,
$\mathrm{CH}-\mathrm{OH}), 2.32\left(\mathrm{~m}, 2 \mathrm{H}, \mathrm{CO}-\mathrm{CH}_{2}\right), 1.62\left(\mathrm{~m}, 2 \mathrm{H}, \mathrm{CO}-\mathrm{CH}_{2}-\mathrm{CH}_{2}\right)$, 1.28 (br s, chain $\mathrm{CH}_{2}$ ), 2.18 and $2.03\left(\mathrm{~m}, 2 \mathrm{H}\right.$, each for $\mathrm{CH}_{2^{-}}$ $\left.\mathrm{CH}=\mathrm{CH}-\mathrm{CH}_{2}\right), 1.30\left(\mathrm{~m}, 2 \mathrm{H}, \mathrm{CH}_{2} \mathrm{CH}_{3}\right), 0.90$ (distorted t, $3 \mathrm{H}$, terminal $\left.\mathrm{CH}_{3}\right),{ }^{13} \mathrm{C} \mathrm{NMR}\left(\mathrm{CDCl}_{3}\right) \delta(\mathrm{ppm}): 173(\mathrm{C}=\mathrm{O}), 152.2$ $(\mathrm{N}=C-\mathrm{N}), 139.0(\mathrm{~N}-C=\mathrm{C}), 140.0\left(\mathrm{O}_{2} \mathrm{~N}-C\right), 34.6\left(\mathrm{~N}-\mathrm{CH}_{2}\right), 62.4$ $\left(\mathrm{N}-\mathrm{CH}_{2}-\mathrm{CH}_{2}\right), 33.9\left(\mathrm{CO}-\mathrm{CH}_{2}\right), 24.5\left(\mathrm{CO}-\mathrm{CH}_{2}-\mathrm{CH}_{2}\right), 29.1$, 29.4, 29.0, 25.3, 34.0 (C-8), 60.4 (C-9), 61.2 (C-10), 45.6 C11), 65.6 (C-12), 34.6 (C-13), 24.7, 28.9, 30.7, 25.5, 14.3, 12.6, ESI-MS $m / z:\left[\mathrm{M}^{+}+1\right] 612.41$.

\section{RESULTS AND DISCUSSION}

The synthesis of long chain fatty acid alkenoates of metronidazole (5-8) and their tetrazole derivatives (5a-7a) major products along with minor products $(\mathbf{5 b} \mathbf{b}-\mathbf{7 b})$ was performed in a manner as outlined in Schemes I-III.

In the IR spectra of fatty alkenoates 5-8, the appearance of characteristic band in the region of $1739-1736 \mathrm{~cm}^{-1}$ confirmed the presence of an ester carbonyl group. The ${ }^{1} \mathrm{H}$ NMR spectra of fatty alkenoates were more informative regarding the structures of 5-8. In addition to the normal signals of fatty ester other characteristic signals were observed in the range of $\delta 7.81-7.93(\mathrm{~s}, 1 \mathrm{H}), \delta 2.40-2.49(\mathrm{~s}, 3 \mathrm{H}$, ) assignable to imidazole ring protons. The signals appeared in the range of $\delta 4.54-$ $4.63(\mathrm{t}, 2 \mathrm{H})$ and $\delta 4.32-4.37(\mathrm{t}, 2 \mathrm{H})$ were attributed to methylene protons $\left(\mathrm{N}-\mathrm{CH}_{2}-\mathrm{CH}_{2}\right)$. These data confirmed the presence of imidazole ring in the fatty alkenoates 5-8. The structures of all these compounds were further confirmed by ${ }^{13} \mathrm{C}$ NMR spectra. A characteristic signal for the ester carbonyl group appeared in the range of $\delta 173.0-173.8$. The signals at $\delta 150.7$ $154.2, \delta 138.5-140.5$ and $\delta 133.0-138.2$ further confirmed the presence of imidazole ring in fatty alkenoates 5-8. Assignment of selected characteristic IR bands provides significant indications for the formation of the $\alpha$-bromo-5'-methyl tetrazole derivatives (5a-7a). All the compounds showed sharp bands in the region 1245-1240 and 984-982 $\mathrm{cm}^{-1}$ which confirmed the formation of tetrazole ring. In addition, the absorption bands at $665-668 \mathrm{~cm}^{-1}$ were attributed to the bromo group, which also confirm the formation of desired $\alpha$-bromo-5'-methyl tetrazole derivatives. The ${ }^{1} \mathrm{H}$ NMR spectrum of compound $\mathbf{5 a}$ was also in agreement with the formation of a tetrazole ring as the chemical shift of $\mathrm{C}-10$ proton has shifted up field from $\delta$ 5.82 to $\delta 4.65$ due to the ring formation. Two methylene protons (Hz, HE) attached to bromine are magnetically non-equivalent by virtue of their different stereochemistry, therefore they have different chemical shift in their ${ }^{1} \mathrm{H}$ NMR spectrum and appeared at $\delta 3.90(\mathrm{~s}, 1 \mathrm{H}, \mathrm{HzC}-\mathrm{Br})$ and $\delta 3.8$ (dist. d, $1 H, \mathrm{HEC}-\mathrm{Br}, J=$ $3.0)$, respectively.

In addition to the normal signals of fatty acid ester (5) other characteristic signal was observed at $\delta 2.51$ for methyl protons of tetrazole ring. These data confirmed the structure of $\mathbf{5 a}$ which was further supported by its ${ }^{13} \mathrm{C}$ NMR studies. The signals at $\delta 45.5, \delta 34.8$ and 150.1 were attributed to $\mathrm{CH}-$ $\mathrm{N}, \mathrm{CH}_{2}-\mathrm{Br}$ and $\mathrm{N}-\mathrm{C}=\mathrm{N}$, respectively indicating the presence of bromo group and tetrazole ring respectively in $\mathbf{5 a}$. The signals due to the imidazole ring and fatty ester resonate at their usual position. Similarly other compounds were characterized from their spectral data. 


\section{Conclusion}

Some long chain fatty alkenoates of 1-[2-hydroxyethyl]2-methyl-5-nitroimidazole and their novel tetrazole derivatives were synthesized. The in vitro antiamicrobial activity was examined by agar well diffusion method ${ }^{30}$. The results showed that all compounds exhibited promising inhibitory action against both the groups of bacteria and two strains of fungus (Tables 1-3).

\begin{tabular}{|c|c|c|c|c|}
\hline \multicolumn{5}{|c|}{$\begin{array}{c}\text { TABLE-1 } \\
\text { ANTIBACTERIAL ACTIVITY OF LONG CHAIN FATTY } \\
\text { ALKENOATES OF METRONIDAZOLE } \\
(\mathbf{5 - 8 )} \text { THEIR TETRAZOLE DERIVATIVES }(\mathbf{5 a - 7 a )}\end{array}$} \\
\hline \multirow{3}{*}{ Comp. } & \multicolumn{4}{|c|}{ Diameter of zone of inhibition (mm) } \\
\hline & \multicolumn{2}{|c|}{ Gram-positive bacteria } & \multicolumn{2}{|c|}{ Gram-negative bacteria } \\
\hline & S. aureus & B. subtilis & S. typhimurium & E. coli \\
\hline 5 & 18.2 & 17.4 & 16.2 & 16.1 \\
\hline 6 & 20.2 & 19.5 & 20.2 & 12.7 \\
\hline 7 & 26.9 & 20.6 & 19.5 & 19.7 \\
\hline 8 & 22.2 & 17.2 & 18.2 & 19.5 \\
\hline $5 \mathbf{a}$ & 16.2 & 14.5 & 16.6 & 15.2 \\
\hline $\mathbf{6 a}$ & 18.5 & 20.2 & 18.3 & 18.2 \\
\hline $7 \mathbf{a}$ & 19.5 & 18.4 & 16.2 & 16.4 \\
\hline Chloramph. & 25.5 & 22.5 & 18.2 & 20.2 \\
\hline DMSO & - & - & - & - \\
\hline
\end{tabular}

\begin{tabular}{ccccc}
\multicolumn{5}{c}{ TABLE-2 } \\
& $\begin{array}{c}\text { MINIMUM INHIBITORY CONCENTRATION } \\
\text { (MIC) OF COMPOUNDS (5-8) AND }(\mathbf{5 a - 7 a )})\end{array}$ \\
\hline \multirow{2}{*}{ Compounds } & \multicolumn{4}{c}{ Minimum inhibitory concentration (MIC) $(\mu \mathrm{g} / \mathrm{mL})$} \\
\cline { 2 - 5 } & S. aureus & B. subtilis & S. typhimurium & E. coli \\
\hline $\mathbf{5}$ & 45 & 54 & 43 & 36 \\
$\mathbf{6}$ & 25 & 25 & 29 & 56 \\
7 & 35 & 38 & 26 & 32 \\
$\mathbf{8}$ & 30 & 28 & 25 & 24 \\
$\mathbf{5 a}$ & 52 & 28 & 35 & 28 \\
$\mathbf{6 a}$ & 60 & 32 & 28 & 25 \\
7a & 72 & 24 & 26 & 34 \\
Chloramph. & 30 & 30 & 30 & 30 \\
\hline
\end{tabular}

\begin{tabular}{ccc|cc}
\multicolumn{5}{c}{ TABLE-3 } \\
\multicolumn{4}{c}{$\begin{array}{c}\text { ANTIFUNGAL ACTIVITY OF LONG CHAIN FATTY } \\
\text { ALKENOATES OF METRONIDAZOLE (5-8) THEIR }\end{array}$} \\
& TETRAZOLE DERIVATIVES (5a-7a) \\
\hline \multirow{3}{*}{ Compound } & \multicolumn{2}{c}{$\begin{array}{c}\text { Diameter of zone of } \\
\text { inhibition (mm) }\end{array}$} & \multicolumn{2}{c}{ MIC $(\mu \mathrm{g} / \mathrm{mL})$} \\
\cline { 2 - 5 } & C. albicans & B. subtilis & C. albicans & B. subtilis \\
\hline $\mathbf{5}$ & 10.2 & 12.2 & 250 & 205 \\
$\mathbf{6}$ & 16.8 & 14.5 & 122 & 255 \\
$\mathbf{7}$ & 20.8 & 19.8 & 60 & 54 \\
$\mathbf{8}$ & 20.5 & 17.6 & 72 & 63 \\
$\mathbf{5 a}$ & 18.0 & 17.9 & 215 & 258 \\
$\mathbf{6 a}$ & 20.0 & 16.5 & 83 & 122 \\
$\mathbf{7 a}$ & 20.2 & 18.2 & 124 & 76 \\
Ketoconazole & 22.5 & 22.5 & 100 & 100 \\
DMSO & - & - & - & - \\
\hline
\end{tabular}

Positive control: Ketoconazole and negative control (DMSO) measured byHalo zone test (unit, $\mathrm{mm}$ )
2-(2-methyl-5-nitro-1H-imidazol-1-yl)-ethyl-threo-12hydroxy-9/10-bromo-10/9-(5'-methyl-1H-tetrazol-1-yl) octadecanoate $(\mathbf{7 a})$ was found most active $\left(\mathrm{IC}_{50}=24.6 \mathrm{mM}\right)$ antimicrobial agent among all the synthesized compounds.

\section{ACKNOWLEDGEMENTS}

This work was supported by Department of Science and Technology, Government of India, SERC (Grant no. SR/FT/ CS-027/2008), New Delhi, India.

\section{REFERENCES}

1. M. Grare, M. Mourer, S. Fontanay, J.-B. Regnouf-de-Vains, C. Finance and R.E. Duval, J. Antimicrob. Chemother, 60, 575 (2007).

2. L. Labanauskas, V. Kalcas, E. Udrenaite, P. Gaidelis, A. Brukstus and V. Dauksas, Pharmazie, 56, 617 (2001).

3. T. Onkol, B. Cakir, M. Sahin and F., Turk. J. Chem., 28, 461 (2004).

4. S. Schenone, O. Bruno, A. Ranise, F. Bondavalli, W. Filippelli, G. Falcone, L. Giordano and M.R. Vitelli, Bioorg. Med. Chem., 9, 2149 (2001).

5. M. Gokce, B. Cakir, K. Erol and M.F. Sahin, Arch. Pharm., 334, 279 (2001).

6. J.J. Baldwin, E.L. Engelhardt, R. Hirschmann, G.S. Ponticello, J.G. Atkinson, B.K. Wasson, C.S. Sweet and A. Scriabine, J. Med. Chem., 23, 65 (1980)

7. A. Varvaresou, A. Tsantili-Kakoulidou, T. Siatra-Papastasikoudi and E. Tiligada, Arzneimittelforschung, 50, 48 (2000).

8. A. Foroumadi, M. Mirzaei and A. Shafiee, Pharmazie, 56, 610 (2001).

9. M.G. Mamolo, V. Falagiani, D. Zampieri, L. Vio and E. Banfi, Farmaco, 56, 587 (2001).

10. H. Chen, Z. Li and Y. Han, J. Agric. Food Chem., 48, 5312 (2000).

11. X.J. Zou, G.Y. Jin and Z.X. Zhang, J. Agric. Food Chem., 50, 1451 (2002).

12. X.J. Zou, L.H. Lai, G.Y. Jin and Z.K. Zhang, J. Agric. Food Chem., 50, 3757 (2002).

13. A. Rauf and H. Parveen, Indian J. Chem., 44B, 1273 (2005).

14. F. Clerici, D. Pocav, M. Guido, A. Lochi, V. Perline and M. Brufani, J. Med. Chem., 44, 931 (2001).

15. A. Rauf, M.R. Banday and R.M. Matto, Acta Chim. Slov., 55, 448 (2008).

16. S.M. Ahmed, F. Ahmad and S.M. Osman, J. Am. Oil Chem. Soc., 62, 1578 (1985).

17. R.S. Upadhayaya, S. Jain, N. Sinha, N. Kishore, R. Chandra and S.K. Arora, Eur. J. Med. Chem., 39, 579 (2004).

18. S.A.F. Rostom, H.M.A. Ashour, H.A. Abd El Razik, A.H. Abd El Fattah and N.N. El Din, Bioorg. Med. Chem., 17, 2410 (2009).

19. S.N. Fedosov, L. Berglund, E. Nexø and T.E. Petersen, J. Organomet. Chem., 692, 1234 (2007).

20. E.-S.M. Sherif, R.M. Erasmus and J.D. Comins, Corros. Sci., 50, 3439 (2008).

21. H.P. Kavitha and N. Balasubramanian, Indian J. Chem. Technol., 9, 361 (2002).

22. K.L. Kees, R.S. Cheeseman, D.H. Prozialeck and K.E. Steiner, J. Med. Chem., 32, 11 (1989).

23. R.I. Ishmetova, G.L. Rusinov, M.A. Kravchenko, D.G. Beresnev, V.G. Kitaeva, E.I. Tolstykh, V.A. Sokolov and O.N. Chupakhin, J. Pharm. Chem., 34, 416 (2000).

24. P.F. Iqbal, H. Parveen, A.R. Bhat, F. Hayat and A. Azam, Eur. J. Med. Chem., 44, 4747 (2009).

25. H. Parveen, F. Hayat, S. Mukhtar, A. Salahuddin, A. Khan, F. Islam and A. Azam, Eur. J. Med. Chem., 46, 4669 (2011).

26. H. Parveen, F. Hayat, A. Salahuddin and A. Azam, Eur. J. Med. Chem., 45, 3497 (2010).

27. A. Hassner and V. Alexanian, Tetrahedron Lett., 19, 4475 (1978).

28. F.D. Gunstone, J. Chem. Soc., 1611 (1954).

29. A. Hassner and F.W. Fowler, J. Org. Chem., 33, 2686 (1968).

30. C. Azoro, World J. Biotechnol., 3, 347 (2002). 